\title{
Conhecimento sobre a doença e expectativas do tratamento em familiares de pacientes no primeiro episódio psicótico: um estudo transversal
}

\author{
Rita Regina Fabri Cabral ${ }^{\star}$ \\ Ana Cristina Chaves**
}

\section{INTRODUÇÃO}

Uma revisão histórica e conceitual da relação entre a família e as doenças mentais graves revela que, na década de 1950, as teorias sistêmicas associavam o relacionamento familiar disfuncional com o início da doença ${ }^{1}$. $O$ objetivo principal da terapia familiar etiologicamente orientada era alterar o sistema familiar através de mudanças no comportamento e no modelo de comunicação dos familiares ${ }^{1,2}$. A partir do final da década de 1970 , as teorias e terapias sistêmicas ligadas à esquizofrenia entraram em declínio por vários motivos, entre eles a decepção com os resultados esperados, mas principalmente porque os familiares sentiam-se sobrecarregados e transformados em "bodes expiatórios" para a causa da doença $a^{2,3}$.

Paralelamente às teorias sistêmicas,

\footnotetext{
* Programa Episódio Psicótico (PEP), Universidade Federal de São Paulo (UNIFESP-EPM). Psiquiatra assistente do Programa Episódio Psicótico (PEP) do Departamento de Psiquiatria da UNIFESP-EPM.

** Programa Episódio Psicótico (PEP), Universidade Federal de São Paulo (UNIFESP-EPM). Doutora. Coordenadora do Programa Episódio Psicótico (PEP) do Departamento de Psiquiatria da UNIFESP-EPM.
}

psiquiatras e sociólogos ingleses estudavam a evolução de pacientes tratados na comunidade após longos períodos de hospitalização². Na década de 1960, desenvolveram a entrevista familiar de Camberwell (EFC) e o construto emoção expressa (EE) para investigar os componentes do envolvimento familiar e também como o ambiente interpessoal poderia influenciar o curso da doença, e não determinar seu início ${ }^{4,5}$.

$\mathrm{Na}$ década de 1980, um grupo de estudiosos seguiu em uma direção diferente dos teóricos sistêmicos ${ }^{2}$. Baseando-se nos estudos de EE e no modelo etiológico de Zubin \& Spring 6 , que integra estresse e vulnerabilidade, desenvolveram uma nova abordagem para familiares de pacientes com esquizofrenia ${ }^{7}$. Tinham como objetivo ajudar as famílias que se sentiam estressadas ao cuidar de seu parente. Propunham sessões educativas sobre a natureza do transtorno e seu tratamento, seguidas por sessões voltadas especificamente para como resolver dificuldades interpessoais e problemas práticos ${ }^{7}$. Em resumo, objetivavam ajudar a diminuir a alta emoção expressa e aumentar a 
capacidade dos membros da família de cuidar e reabilitar o seu parente doente ${ }^{2}$.

As abordagens influenciaram-se mutuamente, e os últimos 20 anos testemunharam o crescimento de uma variedade de estratégias com métodos e objetivos diferentes ${ }^{1}$. Porém, em geral, incluem dois componentes principais: educação dos membros da família sobre o transtorno e apoio e orientação para pacientes e familiares por um período prolongado após um episódio ${ }^{2,8-11}$.

Atualmente, enfatiza-se a necessidade de intervenções precoces, que envolvam uma combinação de medicação e intervenção psicossocial vigorosa. O grupo-alvo são os jovens, e o objetivo das intervenções é prevenir ou limitar o comprometimento mental, psicológico e social após o início do primeiro episódio psicótico ${ }^{12-14}$. Nesse modelo de intervenção, torna-se necessário estabelecer uma aliança terapêutica de colaboração logo nos estágios iniciais, para poder direcionar pacientes e familiares aos aspectos positivos no enfrentamento da doença ${ }^{15}$. Contudo, estudos de intervenção familiar com pacientes no primeiro episódio psicótico são escassos, e diferenças importantes no critério de definição para primeiro episódio psicótico e nas intervenções utilizadas dificultam a comparação entre eles ${ }^{3}$.

Apesar de existir um consenso de que a intervenção familiar é importante, as mudanças de comportamento são processos complexos que não podem ser reduzidos à simples introdução de novas informações ${ }^{16}$. Além disso, não existe consenso sobre como essas informações devem ser apresentadas ${ }^{17}$, e tampouco está claro por que as intervenções familiares são benéficas ou eficazes ${ }^{17}$, ou qual combinação de ingredientes é "ativa" para promover o sucesso dos programas de intervenção familiar ${ }^{17,18}$.

Examinar o relato dos clientes sobre o que consideram útil ou não nas intervenções pode ajudar a esclarecer o processo subjacente e seu impacto terapêutico ${ }^{17}$. Pode ajudar também a determinar as bases de um programa psicoeducacional $^{19}$, verificar que tipo de ajuda os pacientes, familiares e profissionais consideram importantes ${ }^{20}$ e o grau de satisfação com os serviços de atendimento ${ }^{21}$. Conclusões preliminares da pouca literatura existente neste campo sugerem que as necessidades dos pacientes com psicose são diferentes daquelas propostas e elaboradas por profissionais, e que os pacientes com primeiro episódio psicótico e suas famílias estão insatisfeitos com as informações fornecidas ${ }^{20,21}$.
Nesse contexto, o objetivo deste estudo foi investigar o conhecimento dos familiares de pacientes no primeiro episódio psicótico a respeito do transtorno e do tratamento, e determinar sobre o que eles gostariam de conversar em sessões semanais multifamiliares.

\section{MÉTODO}

\section{Descrição do local e da amostra}

Em 1999, pacientes no primeiro episódio psicótico e seus respectivos familiares começaram a ser atendidos num programa ambulatorial denominado Programa Episódio Psicótico (PEP) da Universidade Federal de São Paulo - Escola Paulista de Medicina (UNIFESPEPM).

Os pacientes apresentando um episódio psicótico agudo são encaminhados pelos psiquiatras do Pronto Socorro do Hospital São Paulo. Geralmente chegam ao serviço ambulatorial específico na mesma semana da identificação e são atendidos em um grupo de pacientes. Podem estar ou não em uso de antipsicótico. Se em uso, devem estar com menos de quatro semanas de uso regular.

A definição de primeiro episódio psicótico refere-se a pacientes com idade entre 15 e 45 anos que estejam tendo o seu primeiro contato (na vida) com um serviço especializado de psiquiatria devido ao quadro psicótico atual ${ }^{22}$. 0 critério diagnóstico é amplo, sintomatológico ou sindrômico (os pacientes não têm um diagnóstico psiquiátrico definitivo).

A amostra intencional para o estudo de avaliação incluiu indivíduos adultos, familiares ou pessoas próximas aos pacientes, que mantivessem contato estreito com estes e que pretendiam acompanhá-los nos atendimentos semanais no serviço ambulatorial.

\section{Procedimentos}

Traduziu-se e adaptou-se o questionário apresentado no livro de Anderson et al. ${ }^{7}$, o qual se destina a avaliar a opinião de familiares de pacientes com esquizofrenia após a participação na intervenção proposta pelos autores. Um grupo de duas psiquiatras traduziu o questionário para o português. Em seguida, uma tradutora especializada fez a versão do português para o inglês. As discrepâncias foram discutidas para a tradução definitiva para 0 português e em seguida o questionário foi adaptado para ser utilizado antes da 
intervenção familiar. O questionário final para essa fase contém cinco perguntas com quatro opções de resposta (1. nada, 2. um pouco, 3. moderada ou 4. muito) dirigidas à obtenção de dados sobre o conhecimento percebido a respeito da doença e de seu tratamento, e uma pergunta aberta sobre o que gostariam de conversar nas sessões.

$\mathrm{Na}$ primeira semana de atendimento ambulatorial, o indivíduo identificado como acompanhante ou responsável respondia ao questionário, e na semana seguinte era convidado a freqüentar o grupo semanal multifamiliar desenvolvido especificamente para esse grupo.

Os dados sociodemográficos dos participantes e os dados obtidos pelo questionário foram analisados através do pacote estatístico SPSS-11.0. Para a pergunta aberta, a leitura e releitura sistemática das respostas, realizadas pelas autoras de modo independente, sempre seguidas de discussão, identificaram as variáveis qualitativas apresentadas.

\section{RESULTADOS}

No período compreendido entre janeiro de 2002 e junho de 2003, 63 pacientes acompanhados por 82 familiares iniciaram 0 programa de atendimento. Entre esses, 57 $(69,5 \%)$ responderam ao questionário.

Quanto às características sociodemográficas, $47(82,5 \%)$ eram do sexo feminino e $10(17,5 \%)$ do sexo masculino. A distribuição do parentesco da amostra apresentou uma freqüência maior de mães $(48,2 \%)$ em comparação aos pais $(7,1 \%)$, irmãs ou irmãos $(16,1 \%)$, cônjuges $(12,5 \%)$, amigos (3,6\%) ou outros (12,5\%). Indivíduos com contato diário $(83,9 \%)$ foram mais freqüentes, seguidos por indivíduos com contato semanal $(12,5 \%)$, mensal $(1,8 \%)$ e eventual $(1,8 \%)$. A idade média dos familiares foi de 43,4 anos (DP $=12,3)$.

Nos resultados do questionário, verificamos que os participantes consideraram-se como tendo nenhum (escore 1) ou pouco (escore 2) conhecimento sobre a doença (perguntas 1 e 2), sobre o tratamento utilizado (perguntas 3 e 4), e sobre o atendimento em equipe (pergunta 5). Esses dados estão apresentados na tabela 1 .

Dos 57 participantes, 16 não responderam a pergunta aberta (6) (sobre o que gostariam de conversar). Entre as 41 respostas obtidas, encontramos 10 variáveis. Doze pessoas referiram-se ao aspecto de "como lidar" ou "como agir" com o paciente. Seis pessoas gostariam de "conversar sobre tudo". Seis gostariam de falar sobre o "estado do parente". Quatro pessoas queriam falar sobre a "medicação". Quatro referiram-se ao "tratamento". Três pessoas referiram-se à "causa". Uma pessoa queria "ouvir a opinião dos outros". Três pessoas gostariam de falar sobre "doenças". Uma queria falar sobre as "reações". Uma pessoa queria saber sobre o "futuro". Esses dados estão apresentados na tabela 2 .

\section{DISCUSSÃO}

Esta pesquisa faz parte de um estudo mais amplo, o qual procurou inicialmente conhecer as expectativas dos familiares, e, posteriormente, a opinião dos sujeitos que participaram da intervenção oferecida. Teve como objetivos facilitar a implantação e melhorar a aceitação e a efetividade de uma intervenção psicoeducacional oferecida a familiares de pacientes no primeiro episódio

Tabela 1 - Resultado obtido nas perguntas com opções (conhecimento sobre a doença e o tratamento)

\begin{tabular}{lcccccccc}
\hline Conhecimento sobre & \multicolumn{2}{c}{ 1. Nada } & \multicolumn{2}{c}{ 2. Um pouco } & \multicolumn{2}{c}{ 3. Moderado } & \multicolumn{2}{c}{ 4. Muito } \\
\cline { 2 - 8 } & $N$ & $\%$ & $N$ & $\%$ & $N$ & $\%$ & $N$ & $\%$ \\
\hline 1. "crise psicótica" & 26 & 45,6 & 26 & 45,6 & 5 & 8,8 & - & - \\
2. "sintomas" & 18 & 31,6 & 28 & 49,1 & 9 & 15,8 & 2 & 3,5 \\
3. "tratamento" & 30 & 52,6 & 21 & 36,8 & 6 & 10,5 & - & - \\
4. "medicação" & 28 & 49,1 & 21 & 36,8 & 8 & 14,0 & - & 1,8 \\
5. "equipe" & 32 & 56,1 & 15 & 26,3 & 9 & 15,8 & 1 & 1,8 \\
\hline
\end{tabular}


psicótico. Acredita-se que esses familiares, se precocemente acolhidos, livres da culpa e do estigma, orientados a aceitar a doença e a reconhecer sinais de exacerbação, podem ajudar os pacientes a melhorar seu insight quanto à doença e contribuir para a adesão ao tratamento necessário em cada caso ${ }^{14,15}$. Por esse motivo, as intervenções familiares estão se tornando imprescindíveis nos serviços de saúde mental ${ }^{23,24}$.

Supõe-se que o fato de uma pessoa da família apresentar um transtorno mental afete os familiares de diversas maneiras. Eles podem experimentar uma variedade de emoções culpa, raiva e até mesmo a negação da doença; não se pode esperar que a família seja capaz de fornecer auxílio imediato e apoio ao paciente ${ }^{15}$. Porém, quando os familiares chegam a um serviço especializado em saúde mental, a maior parte apresenta um forte desejo de compreender o problema e quer um tratamento rápido e efica ${ }^{13,15}$.

Os resultados mostraram que a maioria dos acompanhantes eram mães com contato diário com os pacientes, explicável culturalmente, sendo essas as principais cuidadoras de seus filhos em nosso meio.

As respostas mostraram que a maioria considerou não ter nenhum ou ter muito pouco conhecimento sobre o transtorno e o tratamento. Observou-se, no entanto, que muitos deixaram em branco a pergunta aberta

Tabela 2 - Resultados da pergunta aberta: "Sobre o que você gostaria de conversar nos grupos?"

\begin{tabular}{|c|c|c|}
\hline Dimensões* & Exemplos & № $\dagger$ \\
\hline "Em branco & & 16 \\
\hline $\begin{array}{l}\text { "Como lidar" ou "como } \\
\text { agir" }\end{array}$ & $\begin{array}{l}\text { Sobre como lidar com o doente. } \\
\text { Sobre o porquê dessas crises e como lidar com elas. } \\
\text { Receber orientação de como lidar no dia-a-dia com os quadros do paciente. } \\
\text { Como devo agir com as pessoas com essa doença, não sei se devo ser brava ou mansa. }\end{array}$ & 12 \\
\hline "Conversar sobre tudo" & $\begin{array}{l}\text { Tudo o que diz respeito ao tratamento e comportamento dos pacientes. } \\
\text { Esclarecer sobre os assuntos acima e o que posso estar fazendo para ajudá-la. }\end{array}$ & 6 \\
\hline "Estado do parente" & $\begin{array}{l}\text { Sobre o estado do filho. } \\
\text { Sobre o problema do meu filho. }\end{array}$ & 6 \\
\hline "Medicação" & Bem, eu gostaria de conversar sobre a medicação. & 4 \\
\hline "Tratamento" & $\begin{array}{l}\text { Tratamentos em longo prazo e se há eficácia de } 100 \% \text { e os graus dessa doença. } \\
\text { Como a psicose é tratada, como os médicos trabalham em equipe em relação a esse } \\
\text { problema. }\end{array}$ & 4 \\
\hline "Causa" & $\begin{array}{l}\text { Gostaria de saber a causa, como se iniciou, sendo que a pessoa não nasceu com a doença, e } \\
\text { porque apareceu aos } 17 \text { anos e por aí afora. } \\
\text { Saber, conhecer um pouco mais a doença e saber o porque. Esclarecer. }\end{array}$ & 3 \\
\hline "Ouvir outros" & Ouvir a opinião de todos os participantes. & 1 \\
\hline "Doenças" & Sobre "crise psicótica", depressão e convulsão. & 3 \\
\hline "Reações" & Sobre as reações. & 1 \\
\hline "Futuro" & $\begin{array}{l}\text { Gostaria de saber se esta doença tem cura e por quanto tempo deve tomar o remédio e } \\
\text { também se o doente tem condições futuras de ter uma vida normal: trabalhar, família, filhos, } \\
\text { etc. }\end{array}$ & 1 \\
\hline Total & & 57 \\
\hline
\end{tabular}

* Dimensões = variáveis qualitativas das respostas obtidas.

$† N^{\circ}=$ número de ocorrências. 
"sobre o que gostariam de conversar" nas sessões familiares, e que obter conhecimento não foi uma resposta tão freqüente quanto o esperado. Ao invés disso, saber "como agir" ou "como lidar" com o parente enfermo foi um desejo freqüente apontado pelos familiares, provavelmente direcionado à necessidade de gerenciamento da situação de crise diante da psicose, nunca antes vivenciada. Durante a intervenção voltada a esses familiares, ao mesmo tempo em que se fornece educação genérica sobre psicose e seu tratamento, devemos considerar a importância de se estar atento às necessidades específicas de cada caso, uma vez que o diagnóstico de esquizofrenia ou de outro transtorno psicótico não pode ser feito prontamente. Nessa fase inicial, a intervenção pode contribuir no sentido da formulação diagnóstica.

Em um estudo recente, Fresan et al. ${ }^{19}$ tinham como objetivo conhecer o ambiente familiar e determinar as bases de um programa de psicoeducação de acordo com as necessidades dos familiares de pacientes no primeiro episódio. Os resultados mostraram que, embora os participantes relatassem ter conhecimento a respeito da doença ( $6 \%$ sabiam tudo, $12 \%$ muito, $56 \%$ algo, $12 \%$ pouco e $2 \%$ nenhum conhecimento), havia uma percepção de que esse conhecimento era insuficiente para enfrentar uma crise. Em outras palavras, era insuficiente para lidar com a situação e com o familiar enfermo. Segundo o parecer dos autores, o desconhecimento da etiologia estava associado ao sentimento de culpa, e esses dois sentimentos juntos levavam a limitações no manejo da doença ${ }^{19}$.

Embora existam evidências de que psicoeducação familiar reduz as taxas de recaídas e ajuda na recuperação dos pacientes com transtorno mental grave $e^{2,8,9,11}$, aponta-se que o uso rotineiro da intervenção familiar baseada em evidências é extremamente limitado ${ }^{2,8,9}$ e que há insatisfação dos usuários com os programas existentes ${ }^{17,20,21}$. Um dos problemas para sua implantação na prática clínica é como adaptála a um contexto social e cultural específico ${ }^{25}$. Estabelecer o diálogo com uma ampla faixa de sujeitos considerados como colaboradores - gerentes de programas, pacientes, familiares e outros - com atenção não só aos protocolos dos programas, mas também às necessidades específicas locais pode ajudar a esclarecer vários aspectos do processo de adaptação necessário ${ }^{17,20,25}$. importante conhecer as limitações deste estudo. Os familiares e acompanhantes foram todos participantes voluntários, a amostra é pequena, não é de uma área de captação e houve uma perda importante, de aproximadamente $30,5 \%$ (57/82). Todos esses dados dificultam a determinação, de modo mais amplo, da extensão pela qual os resultados podem ser representativos de familiares de pacientes no primeiro episódio psicótico.

\section{REFERÊNCIAS BIBLIOGRÁFICAS}

1. Barbato A, D'Avanzo B. Family interventions in schizophrenia and related disorders: a critical review of clinical trials. Acta Psychiatr Scand. 2000;102:81-97.

2. Fallon $I R H$. Family interventions for mental disorders: efficacy and effectiveness. World Psychiatry. 2003;2(1):20-8.

3. Linszen DH, Birchwood M. The early phase of psychosis and schizophrenia: a critical period for patients, families and the profession. In: Birchwood M, Fowler D, Jackson C, editors. Early intervention in psychosis. Toronto: John Wiley \& Sons; 2000. p. 261-77.

4. Leff J, Vaughn C. Expressed emotion in families. New York: Guilford; 1985. p. 85-96.

5. Mari JJ, Strainer D. An overview of family interventions and relapse on schizophrenia: meta-analysis of research findings. Psycholl Med. 1994;24:565-78.

6. Zubin J, Spring B. Vulnerability - a new view of schizophrenia. J Abnorm Psychol. 1977;56(2):103-26.

7. Anderson CM, Reiss JD, Hogarty GE, editors. Schizophrenia and family. A practitioner's guide to psychoeducation and manager. New York: Guilford; 1986. p. 338-9.

8. Dixon LB, Lehman AF. Family interventions for schizophrenia. Schizophr Bull. 1995;21:631-43.

9. Dixon LB, Adams C, Lucksted A. Update on family psychoeducation for schizophrenia. Schizophr Bull. 2000;26(1):5-20

10. McFarlane WR, Lukens EP. Insight, families and education. In: Amador XF, Anthony SD, editors. Insight and psychosis. Oxford: Oxford University Press; 1998. p. 317-32.

11. Pekkala E, Merinder L. Psychoeducation for schizophrenia. The Cochrane Database of Systematic Reviews 2001, Issue 3. Oxford: Update Software; 2001.

12. Birchwood M, Macmillan F. Early intervention in schizophrenia. Aust N Z J Psychiatry. 1993;27:374-8.

13. McGorry PD. Psychoeducation in first-episode psychosis: a therapeutic process. Psychiatry. 1995;58:313-28.

14. McGorry PD. The concept of recovery and secondary prevention in psychotic disorders. Aust N Z J Psychiatry. 1992;237(26):3-17

15. McGorry PD, Edwards SJ. Intervenção precoce nas psicoses. [Trad. Prof. Dr. Mario Rodrigues Louzâ Neto]. São Paulo: Janssen-Cilag Farmacêutica; 2002. p. 131-55.

16. Melman J. Família e doença mental - repensando a relação entre profissionais de saúde e familiares. São Paulo: Escrituras; 2001.

17. Budd RJ, Hughes ICT. What do relatives of people with schizophrenia find helpful about family intervention? Schizoph Bull. 1997;23(2):341-7.

18. Dixon L. The cup is both half empty and half full. World Psychiatry. 2003;2(1):30-1. 
19. Fresan A, Apiquian R, Loyzaga, Garcia-Anaya M, Ulloa RE, Nicolini H. Recuperación funcional em el primer episodio psicótico. Psiquiatr Salud Integral. 2002;2(2):40-4.

20. de Haan $L$, van Raaij $B$, van den Berg $R$, Jager $M$, Houweling $P$, Stockmann $M$, et al. Preferences for treatment during a first psychotic episode. Eur Psychiatry. 2001;16:83-9.

21. Leavey G, King M, Cole E, Hoar S, Johnson-Sabine. First-onset psychotic illness: patients' and relatives' satisfaction with services. Br J Psychiatry. 1997;170:53-

22. Keshavan MS, Schooler NR. First-episode studies in schizophrenia: criteria and characterization. Schizoph Bull. 1992;18:491-513.

23. Mullen A, Murray L, Happell B. Multiple family group interventions in first episode psychosis: enhancing knowledge and understanding. Int J Ment Health Nurs. 2002;11:225-32.

24. Hogarty GE. Does family psychoeducation have a future? World Psychiatry. 2003;2(1):29-30.

25. Lopez SR, Kipelowicz A. Family interventions for serious mental illness: translating research to practice. World Psychiatry. 2003;2(1):34-5.

\section{RESUMO}

Introdução: Os familiares de pacientes no primeiro episódio psicótico ocupam um lugar importante no tratamento e na recuperação de seus parentes em atendimento.

Objetivo: Avaliar o conhecimento sobre transtorno psicótico e as expectativas de tratamento dos familiares desses pacientes.

Método: Antes de participarem de um grupo multifamiliar, os parentes dos pacientes no primeiro episódio psicótico responderam a um questionário a respeito de seus conhecimentos sobre a doença e de suas expectativas em relação ao tratamento.

Resultados: A amostra constituiu-se de 47 mulheres (82,5\%) e 10 homens (17,5\%), sendo a maioria mães dos pacientes. As respostas mostraram haver nenhum ou pouco conhecimento sobre o que é a crise psicótica, seus sintomas, tratamento e medicação. Em relação ao que gostariam de conversar no grupo de familiares, 16 (28\%) indivíduos não responderam, 12 (21\%) queriam saber como lidar com seu parente doente, seis $(10,5 \%)$ queriam conversar sobre tudo, seis $(10,5 \%)$ queriam entender o problema do parente, $e$ 17 (30\%) queriam conversar sobre a doença e aspectos relacionados.

Discussão: Os resultados mostraram que o conhecimento sobre a doença é escasso e que há interesse, principalmente, em saber como lidar com a situação.

Conclusões: $O$ acolhimento e as informações vão ao encontro das necessidades dos usuários e podem ajudar a melhorar a adesão ao tratamento, a qualidade das relações familiares e a evolução da doença.

Descritores: Terapia familiar, esquizofrenia, transtornos psicóticos.

\section{ABSTRACT}

Introduction: Family members are important in the care and recovery of first-episode psychotic patients.

Objective: To evaluate the knowledge of the disease and the treatment expectations of the relatives of patients at their first psychotic episode.

Method: Before participating in a multi-family group, relatives of first-episode psychotic patients answered a questionnaire concerning their knowledge of the illness and their treatment expectations.

Results: The sample consisted of 47 women (82.5\%) and 10 men (17.5\%), mostly mothers. Answers showed no or little knowledge about the psychotic crisis, its symptoms, treatment and medication. Sixteen (28\%) individuals did not provide an answer for the question about what they would like to discuss in the multi-family group, 12 (21\%) wanted to know how to deal with and care for their ill relative, six $(10.5 \%)$ would like to talk about everything, six $(10.5 \%)$ wanted to understand the patient's problem, and the remaining 17 patients (30\%) wanted to talk about the illness and related subjects.

Discussion: The results show that there is little knowledge about the disease, and that the relatives of first-episode psychotic patients are interested to know how to deal with the situation.

Conclusion: Relatives of first-episode psychotic patients should be offered receptiveness and information. This can have a positive influence on the adherence to treatment, the quality of family relations, and the outcome.

Keywords: Family therapy, schizophrenia, psychotic episodes.

Title: Knowledge of the disease and treatment expectations in relatives of patients with the first psychotic episode: a cross-sectional study

\section{RESUMEN}

Introducción: Los familiares tienen un rol muy importante en el tratamiento y recuperación de los pacientes que están siendo atendidos en el primer episodio psicótico.

Objetivo: Evaluar el conocimiento sobre el trastorno psicótico y las expectativas de tratamiento de los familiares de los pacientes en el primer episodio psicótico.

Método: Antes de participar de un grupo multifamiliar, los parientes de los pacientes en el primer episodio psicótico contestaron a una encuesta sobre sus conocimientos acerca de la enfermedad y sus expectativas con relación al tratamiento.

Resultados: La muestra está formada por 47 mujeres (82,5\%) y 10 hombres (17,5\%), siendo la mayoría, madres de pacientes. Las respuestas indicaron haber poco o ningún conocimiento sobre lo que es un episodio psicótico, o sobre sus síntomas, tratamiento y medicación. Respecto a que les gustaría 
hablar en el grupo familiar, 16 (28\%) individuos no contestaron, 12 (21\%) quisieran saber cómo tratar a su pariente enfermo, seis (10,5\%) quisieran conversar acerca de todo, seis (10,5\%) quisieran entender el problema del pariente y el resto (17 o 30\%) quisieran conversar acerca de la enfermedad y los aspectos relacionados.

Discusión: Los resultados indicaron que el conocimiento acerca de la enfermedad es insuficiente y que hay interés, principalmente, en saber cómo manejar la situación.

Conclusiones: La acogida y las informaciones atienden a las necesidades de los usuarios y pueden ayudar a mejorar la adhesión al tratamiento, la calidad de las relaciones familiares y evolución de la enfermedad.
Palabras clave: Terapia familiar, esquizofrenia, trastornos psicóticos.

Título: Conocimiento sobre la enfermedad y expectativas acerca del tratamiento en los familiares de pacientes en el primer episodio psicótico: un estudio transversal

Correspondência:

Rita Regina Fabri Cabral

Rua Dr. Alceu de Campos Rodrigues, 229/510 - V. N.

Conceição

CEP 04544-000 - São Paulo - SP

Fone/fax: (11) 3845.9228

E-mail: rfcabral@plugnet.com.br

Copyright (c) Revista de Psiquiatria do Rio Grande do Sul - SPRS 